\title{
Tratamiento isotérmico de los aceros aleados al silicio Tipo SAE $92 X^{(\bullet)}$
}

\author{
J.L. Páez ${ }^{(*)}$, F. Fuentes ${ }^{(*)}$ y A. Battagliese ${ }^{(* *)}$ \\ Resumen La composición química de los aceros SAE 92XX es similar a la de la matriz metálica de una fundi- \\ ción esferoidal, por lo que se pensó en someter a un acero de ese tipo a tratamientos de austempering \\ similares a los que se aplican para lograr una ADI (Austempered Ductile Iron), y verificar si se alcan- \\ zaban valores aceptables de plasticidad con elevados valores de resistencia a la tracción para el \\ mismo acero, tal y como sucede con aquellas fundiciones, y comparar dichos resultados con los obte- \\ nidos en el mismo acero con tratamientos convencionales de temple y revenido. Se alcanzaron valo- \\ res que demostraron que, por austempering, se logran excelentes valores de plasticidad, muy superio- \\ res a los alcanzados por temple y revenido para durezas del mismo orden.
}

Palabras clave: Tratamiento térmico. Aceros al silicio. Austempering. ADI.

\section{Isothermal treatment of SAE 92XX Type high silicon steels}

\begin{abstract}
SAE 9260 type steels have silicon and carbon contents similar to those of the ductile iron matrix, and present a bainitic transformation with the same characteristics as ADI (Austempered Ductile Iron). The hypothesis is that excellent mechanical properties can be obtained by means of austempering (in times so short as to be accessible from the industrial point of view), the same as in ADI and even better because it is a rolling material instead of a cast material. It will be compared with the mechanical properties obtained by quenching and tempering at different temperatures.
\end{abstract}

Keywords: Isothermal treatment. Silicon steels. Austempering. ADI.

\section{INTRODUCCIÓN}

El acero SAE 9260 tiene la siguiente composición, en \%: 0,55-0,65 C, 1,80-2,20 Si, 0,7-1,00 Mn, $<0,04$ P y $<0,04 \mathrm{~S}$. La composición de las fundiciones esferoidales, en \%, suele ser: 3-3,5 C, 2-2,5 $\mathrm{Si}$, $0,25-0,35 \mathrm{Mn}, 0,035 \mathrm{P}$ y $0,03 \mathrm{~S}$.

Una fundición esferoidal, calentada a $880-900{ }^{\circ} \mathrm{C}$, pasa al estado austenítico. Enfriada rápidamente a temperaturas del orden de $400-320{ }^{\circ} \mathrm{C}$ y mantenida isotérmicamente entre esas temperaturas, la austenita no se transforma en estructuras bainíticas, como sucede en un acero común, sino que ocurre lo siguiente: la austenita comienza a transformarse en ferrita, y el

(•) La versión definitiva de este trabajo se recibió el día 9 de noviembre de 1995.

(*) Facultad de Ingeniería. Universidad de Buenos Aires. Paseo de Colón 850. CP 1063. Buenos Aires (Argentina).

(**) Laboratorio de Ensayos de Materiales. Escuela Superior Técnica del Ejército. Buenos Aires (Argentina). exceso de carbono que tiene la austenita, que debería precipitar como cementita, no lo hace por la inhibición que provoca la presencia de silicio, y se ve obligado a difundir hacia la austenita restante, enriqueciéndola en carbono y deprimiendo la temperatura de transformación martensítica hasta llegar a suspenderla. Queda entonces la estructura compuesta por ferrita (bainítica) y austenita (estabilizada). Esta estructura, compuesta por constituyentes plásticos, confiere su plasticidad a la aleación (Figs. 1-5).

Pese a ello, con el mantenimiento de la temperatura isotérmica durante un tiempo mayor, el exceso de carbono precipita finalmente como cementita (es decir, se forma bainita superior), con lo que la plasticidad de la estructura vuelve a disminuir (Fig.6).

De ello se deduce que el tratamiento isotérmico precisa un tiempo mínimo, durante el cual el enriquecimiento de carbono llega a estabilizar la austenita, y un tiempo máximo, durante el que el carbono precipita como cementita. Entre ambos, la estructura tendrá buenos valores de plasticidad. 


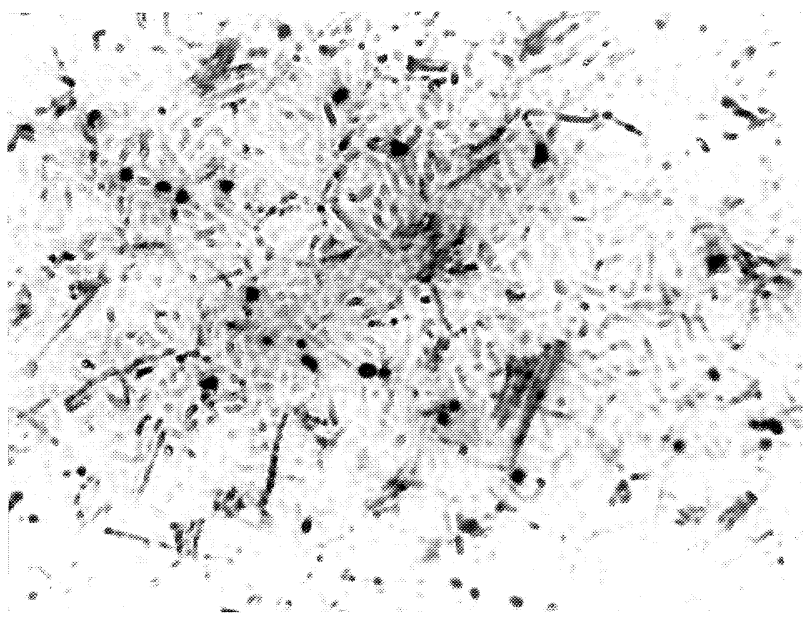

FIG. 1.- Tratamiento de austempering a $370{ }^{\circ} \mathrm{C}$ durante $45 \mathrm{~s}$. Comienzo de la precipitación de agujas de ferrita bainítica. $\times 850$.

FIG. 1.- Austempering at $370{ }^{\circ} \mathrm{C}$ for 45 s. Bainitic ferrite precipitation begins. $\times 850$.

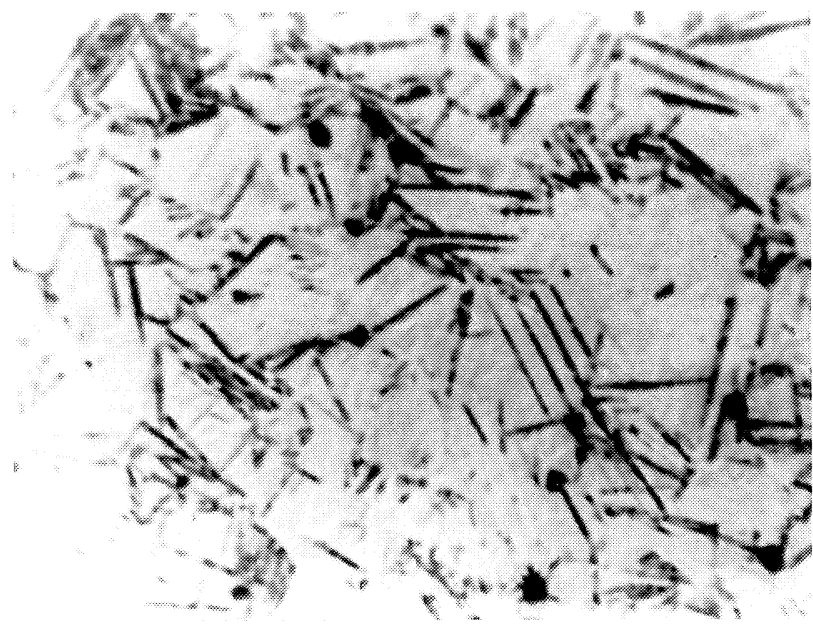

FIG. 2.- Tratamiento de austempering a $370{ }^{\circ} \mathrm{C}$ 80 s. Prosigue la precipitación de agujas de ferrita bainítica. $\times 1.600$.

FIG. 2.- Austempering at $370{ }^{\circ} \mathrm{C}$ for 80 s. The bainitic ferrite precipitation follows its way. $\times 1.600$.

En realidad, para que el proceso sea correcto, se necesitan, además, una temperatura máxima y otra mínima. El límite superior de temperatura será aquél en el cual sea pequeña la diferencia de tiempo entre la estabilización de la austenita y la precipitación de la cementita. El límite inferior de temperatura será aquél en el que la austenita comience a transformarse en bainita inferior, constituyente similar a la bainita superior, pero menos plástico que aquélla, y cuya génesis parece estar controlada por parámetros distintos.

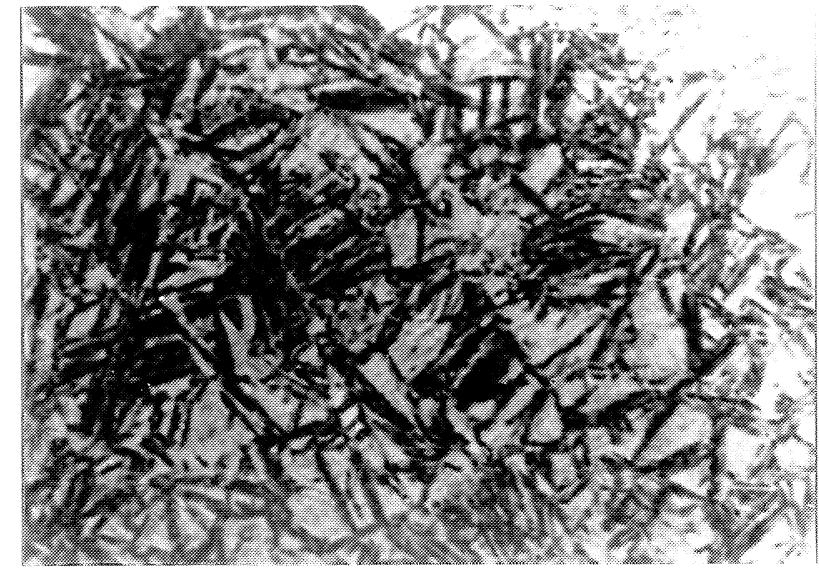

FIG. 3.- Tratamiento de austempering a $370{ }^{\circ} \mathrm{C}$ durante 270 s. Comienza a observarse algo de austenita estabilizada. $\times 1.600$.

FIG. 3.- Austempering at $370{ }^{\circ} \mathrm{C}$ for 270 s. Some retained austenite is already observed. $\times 1.600$

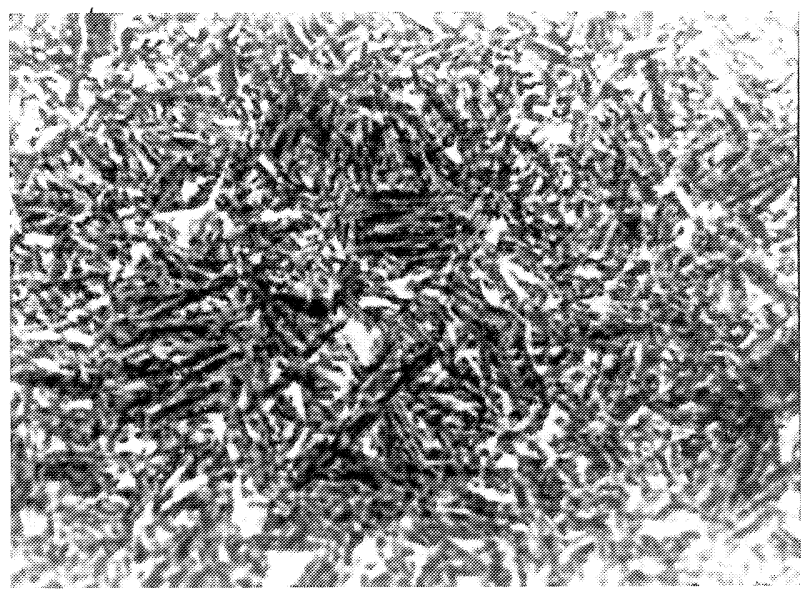

FIG. 4.- Tratamiento de austempering a $370{ }^{\circ} \mathrm{C}$ durante $13 \mathrm{~min}$. Se observa la presencia de mayor cantidad de austenita estabilizada. $\times 850$.

FIG. 4.- Austempering at $370{ }^{\circ} \mathrm{C}$ for $13 \mathrm{~min}$. A more important quantity of retained austenite is observed. $\times 850$.

Es decir, ambos tiempos y ambas isotermas determinan en el diagrama TTT una "ventana", según muestra la figura 7 .

Los puntos dentro de esa "ventana" definen los tratamientos isotérmicos con los cuales se lograrán las estructuras (ferrita bainítica+austenita estabilizada) responsables de buenos valores de plasticidad.

Esta cuestión ha sido muy estudiada (1-4), y configura el fundamento teórico de las ADI (Austempered Ductile Iron o fundición esferoidal austemperada).

Los aceros al silicio Tipo SAE 92XX (9260, 9261 y 9262) tienen contenidos de silicio similares a los de la matriz de una fundición esferoidal y 


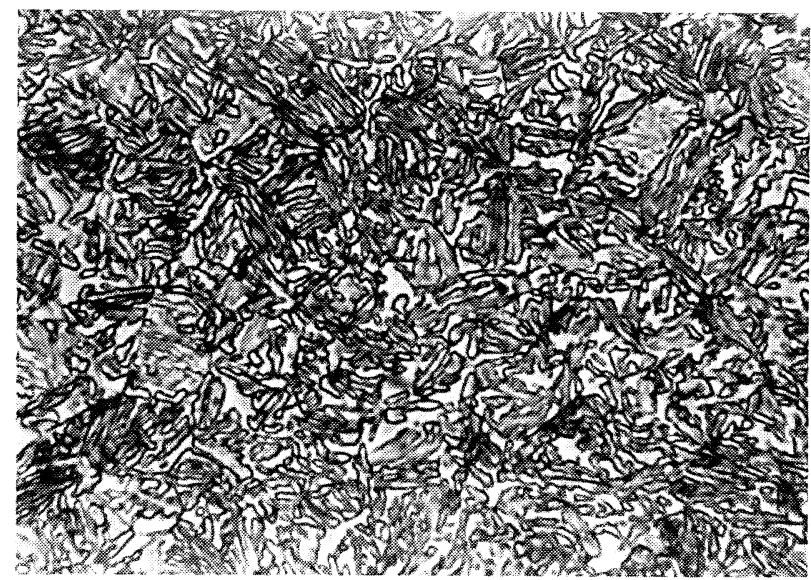

FIG. 5.- Tratamiento de austempering a $370{ }^{\circ} \mathrm{C}$ durante $1 \mathrm{~h}$. Se observa la presencia de ferrita bainítica y austenita estabilizada. $\times 850$.

FIG. 5.- Austempering at $370{ }^{\circ} \mathrm{C}$ for $1 \mathrm{~h}$. The presence of bainitic ferrite and retained austenite is observed. $\times 850$.

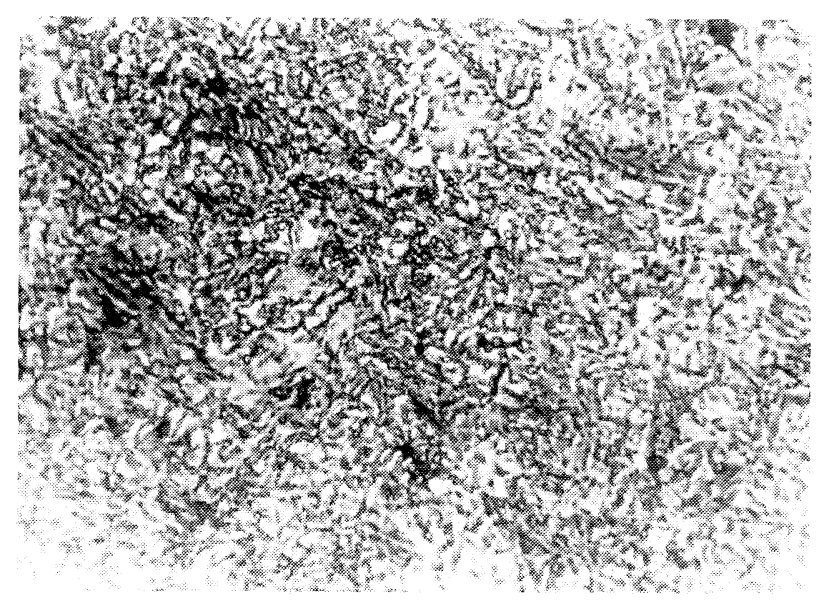

Fig. 6.- Tratamiento de austempering a $370{ }^{\circ} \mathrm{C}$ durante $3 \mathrm{~h}$. Se observa la precipitación de cementita. $\times 850$

FIG. 6.- Austempering at $370{ }^{\circ} \mathrm{C}$ for $3 \mathrm{~h}$. Cementite precipitation is observed. $\times 850$.

presentan una transformación bainítica con las mismas características: esto está estudiado (5) inclusive antes del advenimiento de las ADI. Sin embargo, la bibliografía no se refiere a las propiedades mecánicas que se obtienen de esta manera.

Se pensó en someter estos aceros a tratamientos isotérmicos similares a los de una ADI y comparar los valores obtenidos sobre probetas de tracción con valores obtenidos sobre probetas con tratamientos convencionales de temple y revenido, para durezas del mismo orden, y verificar si la respuesta de los aceros del tipo SAE 92XX al tratamiento isotérmi-

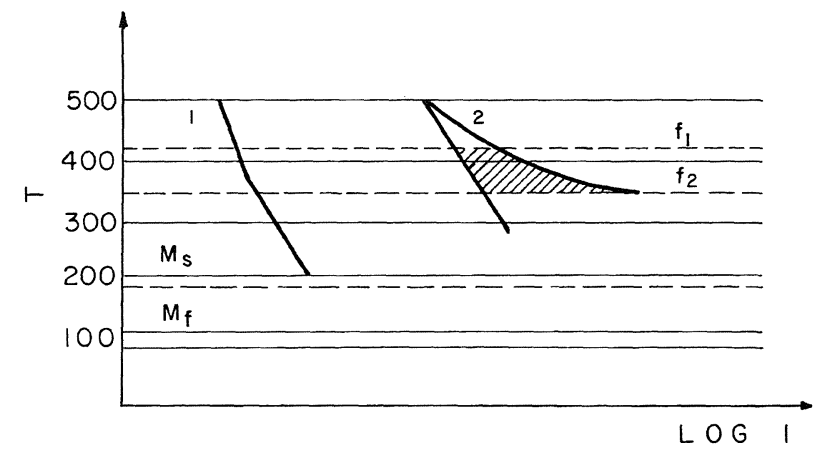

FIG. 7.- La "ventana" en el diagrama TTT.

FIG. 7.- The "window" on TTT diagram.

co es similar a la obtenida en las fundiciones esferoidales.

Para ello, es necesario primero determinar la ubicación de la "ventana" (6) en el correspondiente diagrama TTT.

\section{MATERIALES Y MÉTODOS EXPERIMENTALES}

Para el presente trabajo se utilizaron barras laminadas de acero SAE 9260 y 9262, aptas para forjado, de tamaño de grano núm. 8 (ASTM) y con las siguientes composiciones químicas, en \%:

\section{SAE 9260}

$0,60 \% \mathrm{C}, 1,88 \% \mathrm{Si}, 0,89 \% \mathrm{Mn}, 0,02 \%$ P y 0,007 $\% \mathrm{~S}$

SAE 9262

$0,59 \mathrm{C}, 2,04 \mathrm{Si}, 0,93 \mathrm{Mn}, 0,019 \mathrm{P}, 0,0026 \mathrm{~S}$ y $0,33 \mathrm{Cr}$.

\subsection{Sistema de obtención de la "ventana"}

Se sometió una serie de probetas de aproximadamente $10 \times 10 \times 10 \mathrm{~mm}$, de acero SAE 9260, al siguiente tratamiento: austenización a $870{ }^{\circ} \mathrm{C}$ durante 20 min y posterior enfriamiento en sales de nitrato-nitrito de sodio a diversas temperaturas entre 300 y $420{ }^{\circ} \mathrm{C}$, con permanencia en las mismas durante períodos de tiempo comprendidos entre 15 $\mathrm{s}$ y $6 \mathrm{~h}$ y posterior enfriamiento en agua. La hipótesis de trabajo es la siguiente: si una muestra que hubiese permanecido $n$ segundos a la temperatura isotérmica y posterior enfriamiento en agua no hubiera alcanzado la estabilización de la austenita, se transformaría en martensita con el correspondiente aumento de dureza. Sólo si esa austenita hubiera alcanzado la estabilización no templaría. Por lo tanto, mediciones de dureza (microdureza Vickers $10 \mathrm{~kg}$ ) sobre una secuencia de muestras tratadas a una misma temperatura isotérmica con tiempos variables mostrarían una representación gráfica como la de la figura 8 . 


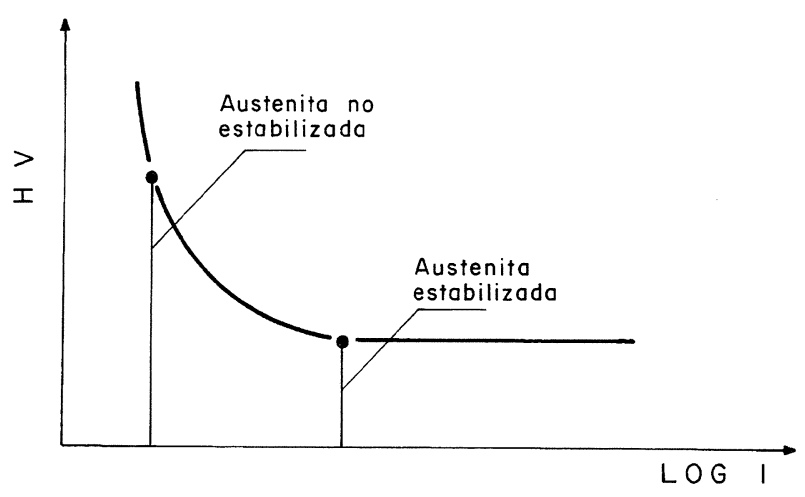

FIG. 8.- Estabilización de la transformación bainítica.

FIG. 8.-Bainitic transformation stabilización.

Cuando la dureza se mantuviera constante, indicaría el tiempo de tratamiento isotérmico para el cual se habría logrado la estabilización de la austenita, es decir, el tiempo mínimo necesario para que en esa isoterma se lograra la estructura (ferrita bainítica+austenita estabilizada).

Así, se lograron las gráficas de las figuras $9 \mathrm{y}$ 10 , que muestran las "ventanas" para los aceros SAE 9260 y SAE 9262.

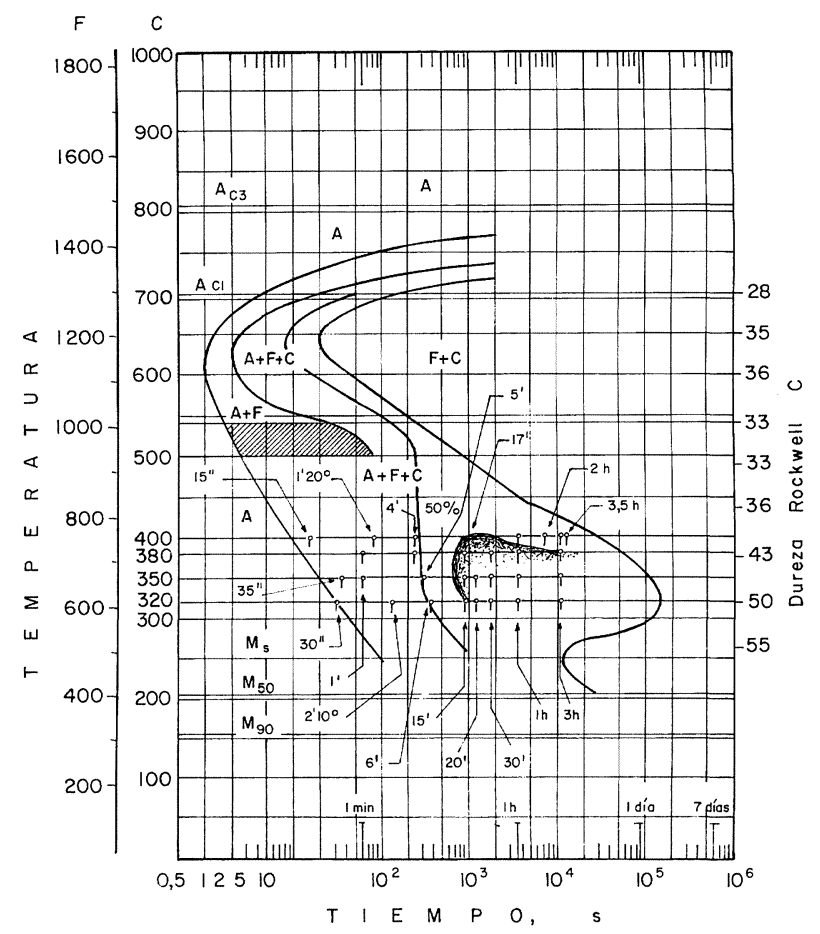

FIG. 9.- "Ventana" del acero SAE 9260 en el diagrama TTT.

FIG. 9.- SAE 9260 steel "window" on TTT diagram.

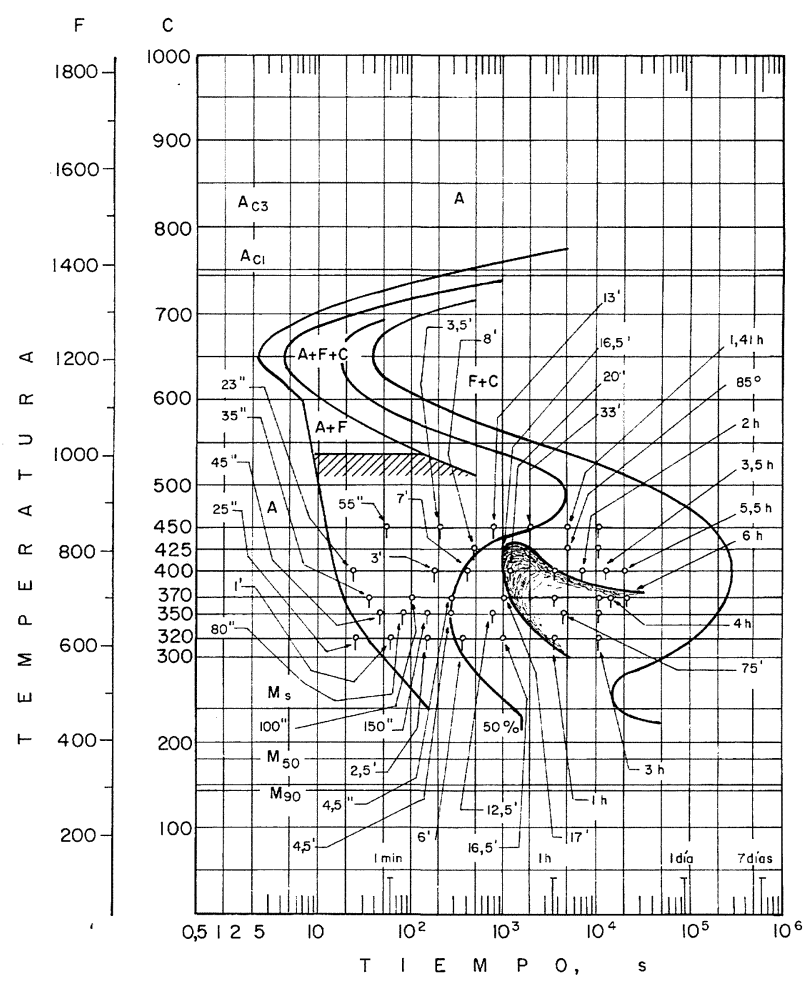

FIG. 10._ "Ventana" del acero SAE 9262 en el diagrama TTT.

FIG. 10.- SAE 9262 steel "window" on TTT diagram.

\subsection{Tratamientos térmicos efectuados sobre probetas de tracción}

Los tratamientos térmicos llevados a cabo consistieron en austenización a $870{ }^{\circ} \mathrm{C}$ durante $20 \mathrm{~min}$ (para todas las probetas).

Las temperaturas y tiempos de austempering fueron:

\section{Serie l:}

Probeta, núm. Temperatura, ${ }^{\circ} \mathrm{C} \quad$ Tiempo, $\mathrm{h}$

$\begin{array}{lll}1-1 & 400 & 1 \\ 1-2 & 370 & 3 \\ 1-3 & 350 & 3 \\ 1-4 & 320 & 3\end{array}$

Finalmente, enfriamiento al agua.

\section{Serie 2:}

Probeta, núm. Temperatura, ${ }^{\circ} \mathrm{C} \quad$ Tiempo, $\mathrm{h}$

$\begin{array}{lll}2-1 & 400 & 1 \\ 2-2 & 370 & 3 \\ 2-3 & 350 & 3 \\ 2-4 & 320 & 5\end{array}$

Finalmente, enfriamiento al agua. 
Serie 3:

Probeta, núm. Temperatura, ${ }^{\circ} \mathrm{C} \quad$ Tiempo, min

$\begin{array}{lll}3-1 & 400 & 20 \\ 3-2 & 370 & 25 \\ 3-3 & 350 & 33 \\ 3-4 & 320 & 60\end{array}$

Finalmente, enfriamiento al agua.

Los tiempos de austempering se seleccionaron de tal manera que, en el diagrama TTT correspondiente a este material, estuviesen dentro de la "ventana".

A la vez, se trataron probetas del mismo material por temple y revenido de acuerdo con el siguiente tratamiento:

Austenización a $870{ }^{\circ} \mathrm{C}$ durante 20 min y enfriamiento en aceite con revenido durante $2 \mathrm{~h}$ a las siguientes temperaturas:

$\begin{array}{cc}\text { Probeta, núm. } & \text { Temperatura, }{ }^{\circ} \mathrm{C} \\ \text { TR-1 } & 600 \\ \text { TR-2 } & 550 \\ \text { TR-3 } & 505 \\ \text { TR-4 } & 480\end{array}$

La dureza obtenida por temple en aceite estaba comprendida entre 62-63 HR C.

\section{RESULTADOS}

Las microestructuras obtenidas a $370{ }^{\circ} \mathrm{C}$ con permanencia en el baño isotérmico durante $1 \mathrm{~h}$, y a $320{ }^{\circ} \mathrm{C}$ durante $3 \mathrm{~h}$, se muestran en las figuras $5 \mathrm{y}$ 11 , respectivamente.

En la primera, se aprecia ferrita y austenita estabilizada, y en la segunda aparecen en la estructura largas agujas obscuras de bainita inferior, responsables de una mayor dureza y de un menor alargamiento plástico.

Se obtuvieron los siguientes valores de dureza, $\mathrm{HR} C$, resistencia a la tracción, $\sigma R$, límite elástico $0,2 \%, \sigma 0,2$, y alargamiento a la rotura, $\delta$ :

$\begin{array}{ccccc}\text { Probeta, núm. } & \text { HR C } & \sigma R, \mathrm{MPa} & \sigma 0,2, \mathrm{MPa} & \delta, \% \\ 1-1 & 37 & 1.380 & 935 & 29,2 \\ 1-2 & 42 & 1.400 & 1.080 & 30,5 \\ 1-3 & 48 & 1.730 & 1.460 & 11,9 \\ & & & & \\ 2-1 & 38 & 1.320 & 910 & 32,2 \\ 2-2 & 40 & 1.390 & 1.070 & 31,7 \\ 2-3 & 43 & 1.510 & 1.230 & 21,1 \\ 2-4 & 47,5 & 1.780 & 1.442 & 13,9 \\ 3-1 & 38 & 1.310 & 899 & 18,4 \\ 3-2 & 43 & 1.480 & 1.080 & 22,2 \\ 3-3 & 45 & 1.580 & 1.210 & 17,5 \\ 3-4 & 51 & 1.780 & 1.450 & 12,9\end{array}$

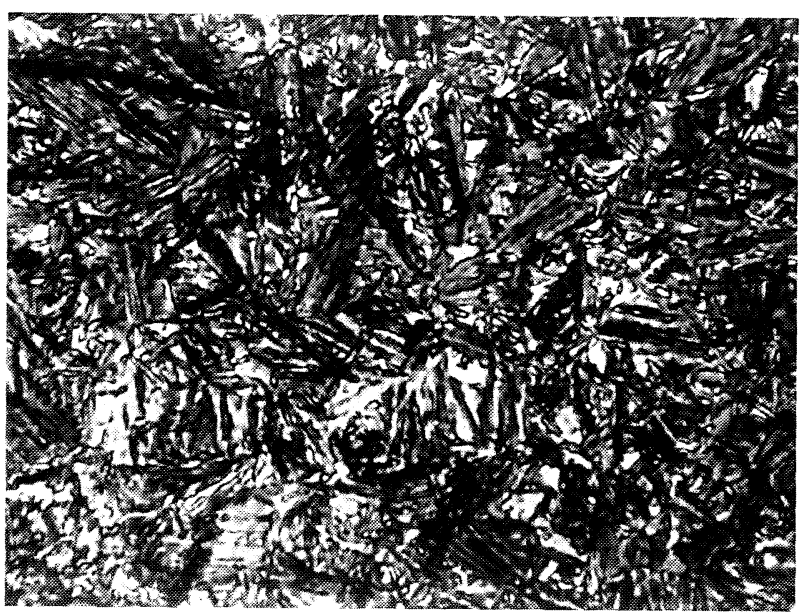

FIG. 11.- Tratamiento de austempering a $320{ }^{\circ} \mathrm{C}$ durante $3 \mathrm{~h}$. Se observa la presencia de bainita inferior. ferrita bainítica y austenita estabilizada $(\times 850)$.

FIG. 11.- Austempering at $320{ }^{\circ} \mathrm{C}$ for $3 \mathrm{~h}$. The presence of lower bainite, bainitic ferrite and retained austenite is observed $(\times 850)$.

$\begin{array}{lllll}\text { TR-1 } & 30 & 1.190 & 1.040 & 14,4 \\ \text { TR-2 } & 42 & 1.390 & 1.240 & 10,8 \\ \text { TR-3 } & 44 & 1.480 & 1.330 & 12,2 \\ \text { TR-4 } & 47 & 1.780 & 1.600 & 8,1\end{array}$

En las figuras 12 a 15 se representan gráficamente los resultados obtenidos. Se aprecia que se ha logrado un alto valor para el alargamiento, que, en el entorno de 40 Rockwell C de dureza, llega a sobrepasar el $30 \%$. En las figuras $1-5$ y 11 se pueden observar las estructuras metalográficas obtenidas.

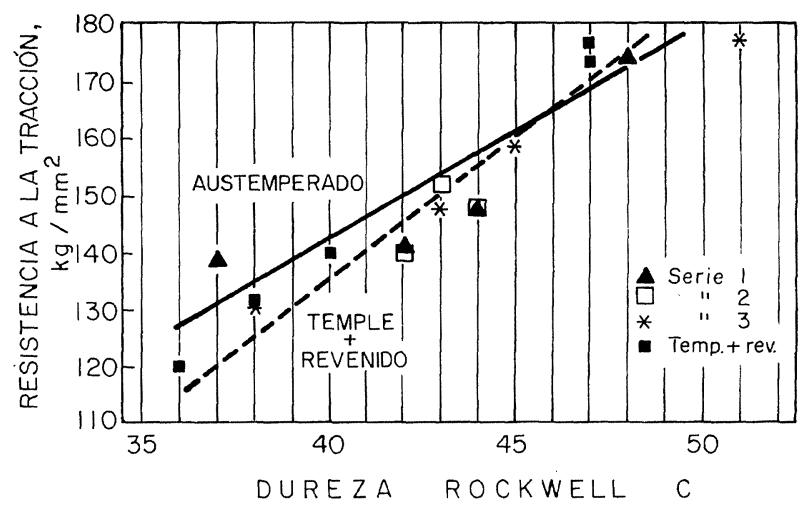

FIG. 12.- Resistencia mecánica obtenida sobre probetas tratadas por austempering y por temple + revenido.

FIG. 12.- Tensile strength relationship between austempered vs. quenched and tempered samples. 


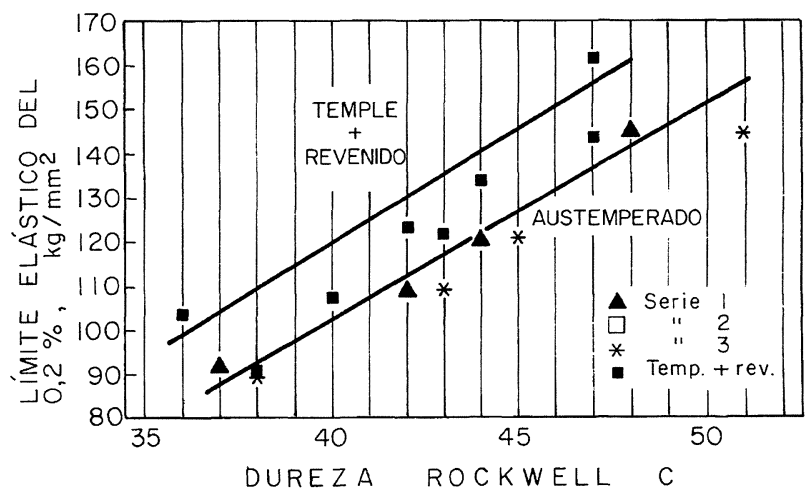

FIG. 13.- Límite elástico obtenido sobre probetas tratadas por austempering y por temple + revenido.

FIG. 13.- Yield strength relationship between austempered and quenched and tempered samples.

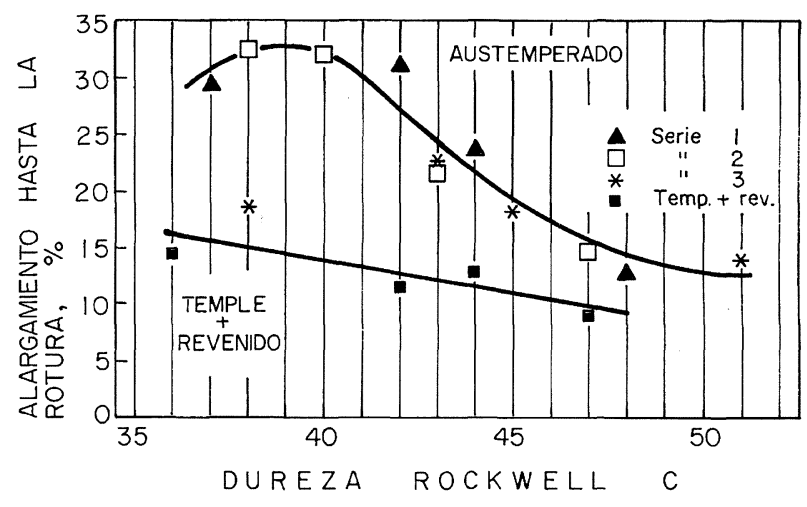

FIG. 14.- Alargamiento obtenido sobre probetas tratadas por austempering y por temple + revenido.

FIG. 14.- Elongation relationship between austempered and quenched + tempered samples.

\section{DISCUSIÓN}

Evidentemente, el tratamiento térmico de austempering, llevado a cabo en aceros aleados al silicio del tipo SAE 9260, les confiere altos valores de resistencia mecánica con muy buenos valores de alargamiento plástico.

Estas propiedades mecánicas son consecuencia de la estructura metalográfica lograda, ferrita bainítica+austenita estabilizada, que se revela como muy superior a la obtenida por temple y revenido.

La estructura mencionada es producto de la alteración de la precipitación bainítica, que resulta inhibida presuntamente por la acción del silicio. Esto determina que el carbono, que debería precipitar desde la austenita en forma de cementita, no lo haga, y difunda en cambio en la propia austenita, estabilizándola por el enriquecimiento en carbono.

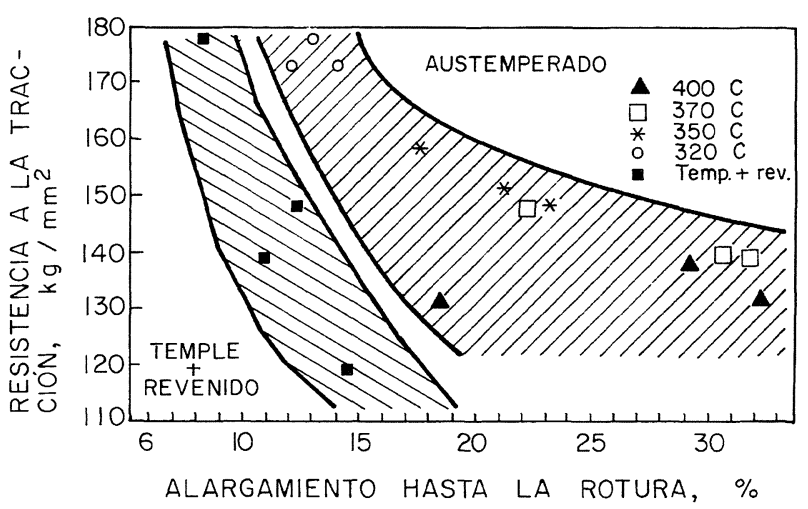

FIG. 15.- Relación entre la resistencia mecánica y el alargamiento obtenida sobre probetas tratadas por austempering y por temple + revenido.

FIG. 15.- Tensile strength vs. elongation relationship between austempered and quenched + tempered samples.

Así, finalmente, la estructura metalográfica queda compuesta por ferrita (bainítica) y austenita estabilizada, ambas fases con buenas propiedades plásticas.

Se debe hacer notar que el tiempo de permanencia en el tratamiento isotérmico es fundamental, porque debe ser lo suficientemente largo como para que el enriquecimiento en carbono de la austenita sea el adecuado para estabilizarla, pero no debe superar cierta concentración que, finalmente, haría precipitar cementita con la consiguiente pérdida de plasticidad. El tiempo mencionado, si bien es prolongado (del orden de $3 \mathrm{~h}$ ), se puede considerar industrialmente adecuado. Esta es una diferencia muy importante si se compara con una transformación isotérmica normal, para la que, de acuerdo con el diagrama TTT, se requieren del orden de $12 \mathrm{~h}$ para una transformación total de la austenita a temperaturas comprendidas entre 300 y $400{ }^{\circ} \mathrm{C}$, lo cual es evidentemente inviable desde el punto de vista industrial. Es decir, que un tratamiento térmico de austempering convencional no sería aplicable en un acero del tipo SAE 9260 como lo es en aceros donde normalmente se lleva a cabo, por ejemplo, en un acero SAE 1070.

Queda demostrado, además, que los aceros del tipo SAE 9260 se comportan de forma similar a como lo hacen las fundiciones esferoidales en los tratamientos isotérmicos ADI.

Obsérvese que, para valores de dureza más elevados, los de alargamiento, si bien son mayores que los logrados con tratamientos de temple y revenido, son sensiblemente menores. Se considera que esto se debe a la precipitación de bainita inferior, a pesar de que algunos autores opinan lo contrario (7). Es 
un detalle muy interesante si se relaciona con lo que sucede con las fundiciones con tratamiento térmico ADI, donde estas estructuras le confieren muy buenas características en cuanto a resistencia al desgaste (8). Quedan entonces abiertas las posibilidades de investigar si con tratamientos isotérmicos de austempering en ese entorno de temperaturas (320 ${ }^{\circ} \mathrm{C}$ ), pueden obtenerse características mecánicas que, además de incluir elevada resistencia con buen alargamiento a la rotura, tengan buena resistencia al desgaste, lo que ampliaría considerablemente el campo de aplicación.

\section{CONCLUSIONES}

El tratamiento isotérmico de austempering aplicado sobre aceros del tipo SAE 9260, revela ser útil para lograr elevada resistencia mecánica combinada con altos valores de plasticidad, muy superiores a los obtenidos con tratamientos térmicos de temple y revenido. Los tiempos de tratamiento del austempering son industrialmente prácticos, si bien éstos deben determinarse previamente con suficiente pre- cisión por ser un factor importante para la obtención de las mencionadas características mecánicas.

\section{REFERENCIAS}

(1) PAEZ, J.L. Mejoramiento de las características mecánicas en fundiciones esferoidales de fabricación normal por tratamientos isotérmicos. Rev. Ingeniería Militar, Núms. 14 y 15. Ejército Argentino. Argentina.

(2) Moore, D.J., Rounds, T.N. y Rundman, K.B. Trans. Am. Foundrymen's Soc., 1985: 705-718.

(3) Rounds, T.N., Rundman, K.B. y Moore, D.J. Trans. Am. Foundrymen's Soc., 1984: 815-840.

(4) Gagne, M. Trans. Am. Foundrymen's Soc., 1984: 801-812.

(5) Le Houllier, R., Bégin, G. y Dubé, A. Metall. Trans., 1987.

(6) Moore, D.J., Rounds, T.N. y Rundman, K.B. Trans. Am. Foundrymen's Soc., 1987.

(7) Sandvick, B.P.J. y Nevalainen, H.P. Met. Technol., Jun. 1981.

(8) ForRest, R.D. Austempered ductile iron (ADI) properties, applications and future potential. Conf. The Iron and Steel Society. Dallas, TX (EE.UU.), Dic., 1986. 